\title{
Induced hypertension for the treatment of acute MCA occlusion beyond the thrombolysis window: case report
} Tanya Bogoslovsky¹, Olli Häppölä ${ }^{1}$ Oili Salonen² ${ }^{2}$ and Perttu J Lindsberg*1,3 Address: ${ }^{1}$ Department of Neurology, Helsinki University Central Hospital, Helsinki, Finland, ${ }^{2}$ Department of Radiology, Helsinki University
Central Hospital, Helsinki, Finland and ${ }^{3}$ Neuroscience Program, Biomedicum Helsinki, Helsinki, Finland

Email: Tanya Bogoslovsky - tanya.bogoslovsky@hus.fi; Olli Häppölä - olli.happola@hus.fi; Oili Salonen - oili.salonen@hus.fi; Perttu J Lindsberg* - perttu.lindsberg@hus.fi

* Corresponding author

Published: 19 December 2006

BMC Neurology 2006, 6:46 doi:10.1 186/147/-2377-6-46
Received: 30 June 2006

Accepted: 19 December 2006

This article is available from: http://www.biomedcentral.com/I47/-2377/6/46

(C) 2006 Bogoslovsky et al; licensee BioMed Central Ltd.

This is an Open Access article distributed under the terms of the Creative Commons Attribution License (http://creativecommons.org/licenses/by/2.0), which permits unrestricted use, distribution, and reproduction in any medium, provided the original work is properly cited.

\begin{abstract}
Background: A minority of stroke patients is eligible for thrombolytic therapy. Small pilot case series have hinted that elevation of incident arterial blood pressure might be associated with a favorable prognosis either in acute or subacute stroke. However, these patients were not considered for thrombolytic therapy and were not followed - up systematically. We used pharmacologically induced hypertension in a stroke patient with middle cerebral artery (MCA) occlusion ineligible for thrombolysis that was followed-up by radiological, clinical and functional outcome assessment.
\end{abstract}

Case presentation: A patient with acute embolic MCA occlusion producing a large, ischemic penumbra confirmed by perfusion CT was treated by induced hypertension with phenylephrine started within $4 \mathrm{~h}$ of admission. Increase in the mean arterial pressure by $20 \%$ led to a reduction of neurological deficit by 3 points on the National Institute of Stroke Scale. MRI and CT scans performed during phenylephrine infusion showed the presence of limited subcortical and cortical infarct changes that were clearly less extensive than the perfusion deficit in the brain perfusion CT at baseline, found in the absence of MCA patency. No complications due to induced hypertension therapy occurred. Moderate functional improvement up to modified Rankin scale 2 at follow up took place.

Conclusion: Induced hypertension in acute ischemic stroke seems clinically feasible and may be beneficial in selected normo- or hypotensive stroke patients not eligible for thrombolytic recanalization therapy.

\section{Background}

Blood pressure (BP) increases after the first minutes of acute cerebrovascular occlusion [1] and decreases significantly from admission to 12 hours after thrombolysis especially in case of adequate recanalization [2]. The physiologic reasons for BP elevation, presumably may represent increased sympathetic autonomic nervous activ- ity mediated by cerebral reticular neuronal networks and the medullary vasomotor center that regulate the tone of resistance of vessels of the body. Due to the failure of auto-regulation of cerebral blood flow (CBF) in the ischemic human brain, the CBF is passively dependent on the mean arterial pressure [3]. Thus, the acutely elevated $\mathrm{BP}$ may help to maintain a vital level of CBF to support the 
existing penumbra, and contribute to the preservation of neurological function. Therefore, hypertension may be a risk marker of poor outcome due to initially large infarction or failed vessel recanalization [2] rather than a causative factor of the acute ischemic stroke [4].

Induced hypertension (IH) is a standard treatment for cerebral ischemia in patients with vasospasm after subarachnoidal hemorrhage. Elevation of BP may improve neurological function and $\mathrm{CBF}$ in subacute ischemic stroke [5], or alleviate the degree of neurologic dysfunction during acute ischemic stroke [6]. Phenylephrineinduced hypertension (PHE-IH) decreased perfusion deficit by $40 \%$ as detected by MRI on the next day [7], suggesting the efficacy of PHE-IH in precluding infarct maturation within the ischemic penumbra.

We report a case where thrombolysis of acute embolic MCA occlusion was not possible due to undocumented onset time of stroke symptoms, but where a large area of cerebral cortex corresponding to ischemic penumbra did not undergo infarction following IH therapy.

\section{Case presentation}

A 72-year right-handed woman with paroxysmal atrial fibrillation (AF), hypertension, coronary artery disease (CAD), asthma and 2 recent transient ischemic attacks was admitted to the emergency department with left hemiparesis and dysarthria that were noticed on awakening $4 \mathrm{~h} 30 \mathrm{~min}$ prior to the admission. She had no anticoagulant therapy. The baseline Barthel index of activities of daily living was 95 and Rankin scale (mRS) was 1 . Neurologic examination revealed mild disorientation, left sensomotor hemiparesis, dysarthria, and visual and tactile neglect. The National Institute of Health Stroke Scale (NIHSS) score was 14 . The mean BP was $150 / 72 \mathrm{mmHg}$, the mean MAP (mean arterial pressure) was $98 \mathrm{mmHg}$ and ECG showed AF.

Immediate brain CT showed a hyperdense right MCA, hypoattenuation of the right lentiform nucleus and insular cortex as well as slight swelling of cortical sulci in the right frontoparietal region. Standard noncontrast CT scanning was performed with a Lightspeed Ultra CT scanner (General Electric Medical Systems; Version $05 \mathrm{MW}$ 14.5.H2_P_M8_G) using the following parameters: 140 $\mathrm{kV}$ (posterior fossa/infratentorial)/ $120 \mathrm{kV}$ (supratentorial), automatic $\mathrm{mA}(80-150 \mathrm{~mA}), 512 \times 512$ image matrix, 23-cm displayed field of view (DFOV), and 5-mm (posterior fossa/infratentorial)/7,5-mm (supratentorial) slice thickness.

In CT perfusion examination, the flow- and mean transit time images showed a broad perfusion deficit in the right MCA region. The blood volume chart, however, showed a clearly less significant defect in this region, consistent with the presence of large ischemic penumbra in the cortical cerebral tissue within the MCA territory (Fig 1, top row). CT angiography documented a thrombus of $1 \mathrm{~cm}$ in length in the right MCA. Some signs of blood flow were detected distally in the right MCA, presumably resulting from collateral circulation (not shown). For the perfusion CT study, 4 adjacent levels were selected at the level of the basal nuclei. Fifty 5-mm CT sections of continuous (cine) scanning $(80 \mathrm{kV}, 200 \mathrm{~mA})$ were obtained at every adjacent level, with a total acquisition time of 50 seconds. Computed tomography was initiated 5 seconds after the intravenous rapid infusion (injection rate of $7 \mathrm{ml} / \mathrm{s}$ ) of 350 $\mathrm{mg} / \mathrm{ml}$ iodinated nonionic contrast material (Iomeron 350, Bracco). The contrast agent was injected into an antecubital vein with a power injector (Medrad).

A diagnosis of a cardio-embolic right MCA occlusion was thus established. Due to the delay in admission and uncertain timing of symptom onset the patient was not eligible for thrombolysis. In attempt to salvage the obviously large ischemic penumbra, induced hypertension (IH) therapy by phenylephrine (PHE) (Neo-Synephrine $0,1 \mathrm{mg} / \mathrm{ml}$; initial dose $0.5 \mathrm{mg} /$ hour)) and crystalloids $3000 \mathrm{ml} /$ day intravenously was initiated 4 hours after the admission. The goal of $20 \%$ MAP augmentation was achieved within 1 hour after the initiation of the PHEinfusion (Figure 1). During the next 4 days the average MAP was maintained at 120 (range 140-91) $\mathrm{mmHg}$ with PHE infusion rate up to $3,5 \mathrm{mg} /$ hour, thereafter PHE was tapered off during 2 days (average MAP 114,5 (range 13399) $\mathrm{mmHg}$ ). During PHE infusion the patient had paroxysmal AF with average heart rate 100 beats $/ \mathrm{min}$. The antihypertensive medication (Athenolol and Lozartan) that the patient had before the admission was stopped at the time of initiation of $\mathrm{IH}$.

Four hours after the initiation of MAP augmentation the gaze deviation and the left leg weakness were improved and NIHSS was 12. Sixteen hours after the MAP augmentation the left facial weakness and hemiparesis were improved, although the patient was still slightly somnolent, and had moderate dysarthria and neglect. The NIHSS improved to 11 . The brain CT and MRI scans performed $21 \mathrm{~h}$ and $26 \mathrm{~h}$ after admission showed the presence of a limited subcortical infarct on the distal region of the right lentiform nucleus and corona radiata. There were only subtle signs of a cortical infarction. Moreover, the extent of the subcortical infarct was substantially smaller than that of the perfusion deficit found in the brain perfusion CT on admission (Fig. 2, bottom row). MRA showed the persistence of the right MCA occlusion (Fig. 2, middle row). 


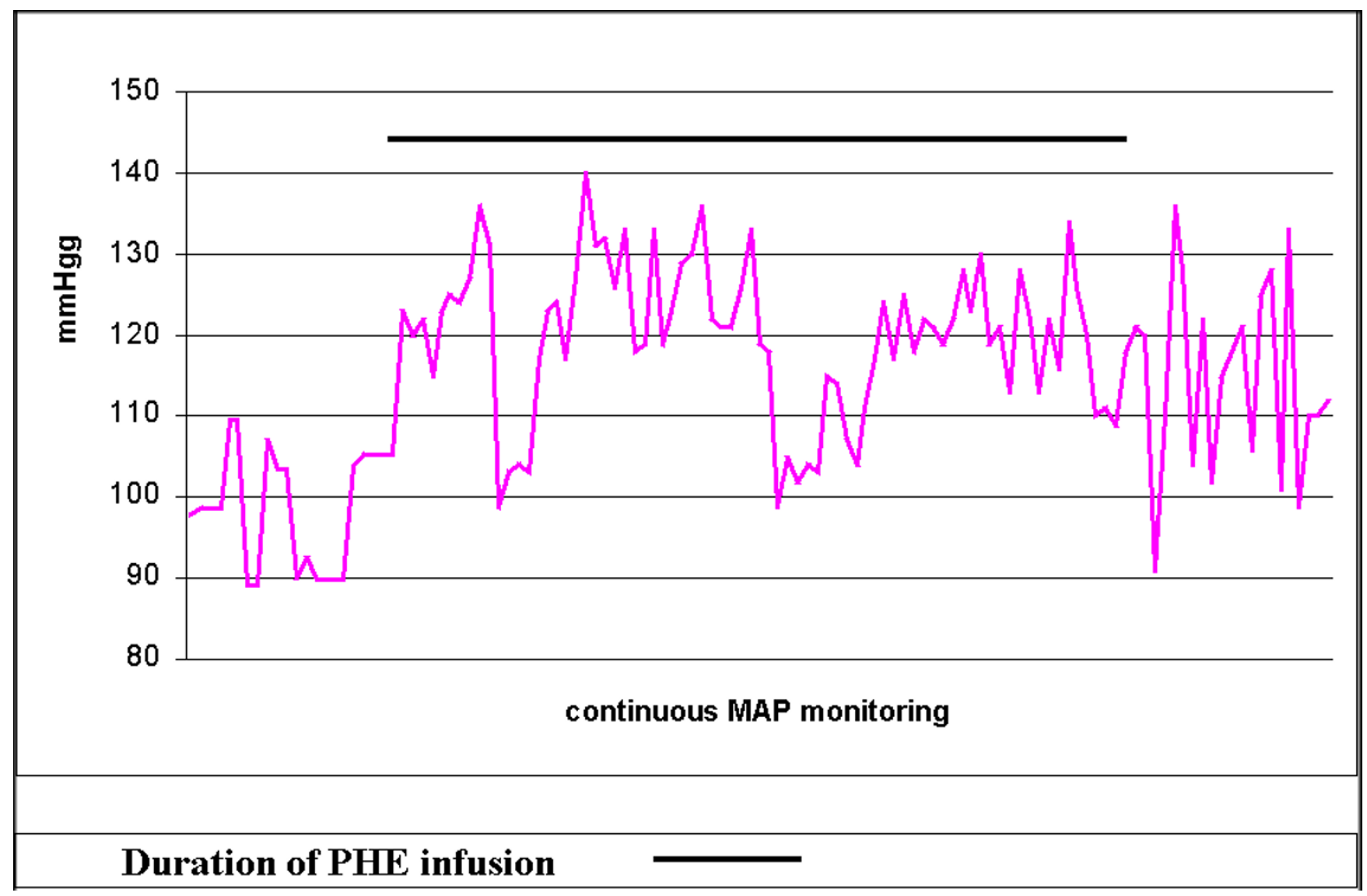

Figure I

Mean MAP on admission, during induced hypertension (days I-5) and tapering off (days 6-7).

During the following days the patient experienced a mild improvement on her level of alertness and in the left hemiparesis, with less significant improvement in the severity of dysarthria and neglect. Antithrombotic treatment with sodium daltreparin followed by warfarin was instituted. When discharged to the rehabilitation hospital, the patient had a NIHSS of 7. At the 3-month control visit she was independent in main activities of daily living, but needed a walking device when strolling outside. The uncontrasted control brain CT showed the previous right MCA infarct without any new signs of ischemia (not shown). NIHSS was 4; Barthel index was 80 and mRS 3. At 10-month interview she lived at home and walked independently, Barthel index was 90 and mRS 2 .

\section{Conclusion}

Many stroke patients are not eligible for thrombolysis due to delay in the admission or unclear timing of symptom onset. Still, there may be a therapeutic window for salvaging of brain tissue through improvement of CBF in the ischemic penumbra, which seems to exist well beyond the sofar approved 3-hour window.
This case study suggests the potential utility of IH in the treatment of acute stroke beyond the conventional thrombolytic treatment window. Studies have documented the existence of an ischemic penumbra up to 24 hours after stroke onset the and therapeutic time window for salvaging brain tissue may be substantially longer [8].

Sparing of the ischemic penumbral brain tissue in the present case was confirmed by the follow-up MRI and supported by the relatively good functional outcome. The spontaneous clot lysis or sudden improvement of collaterals to the occluded MCA territory seems unlikely because the original vascular occlusion as well as collateral flow remained essentially identical on the follow-up MRA. A degree of functional improvement was achieved already acutely upon increasing the average MAP by $20 \%$.

Although we cannot confirm that it was PHE-IH that was crucially responsible for the diversion toward a benign outcome, our past experience of nonrecanalized acute MCA occlusions in elderly patients attests of a much more extensive infarct maturation in the MCA territory, espe- 

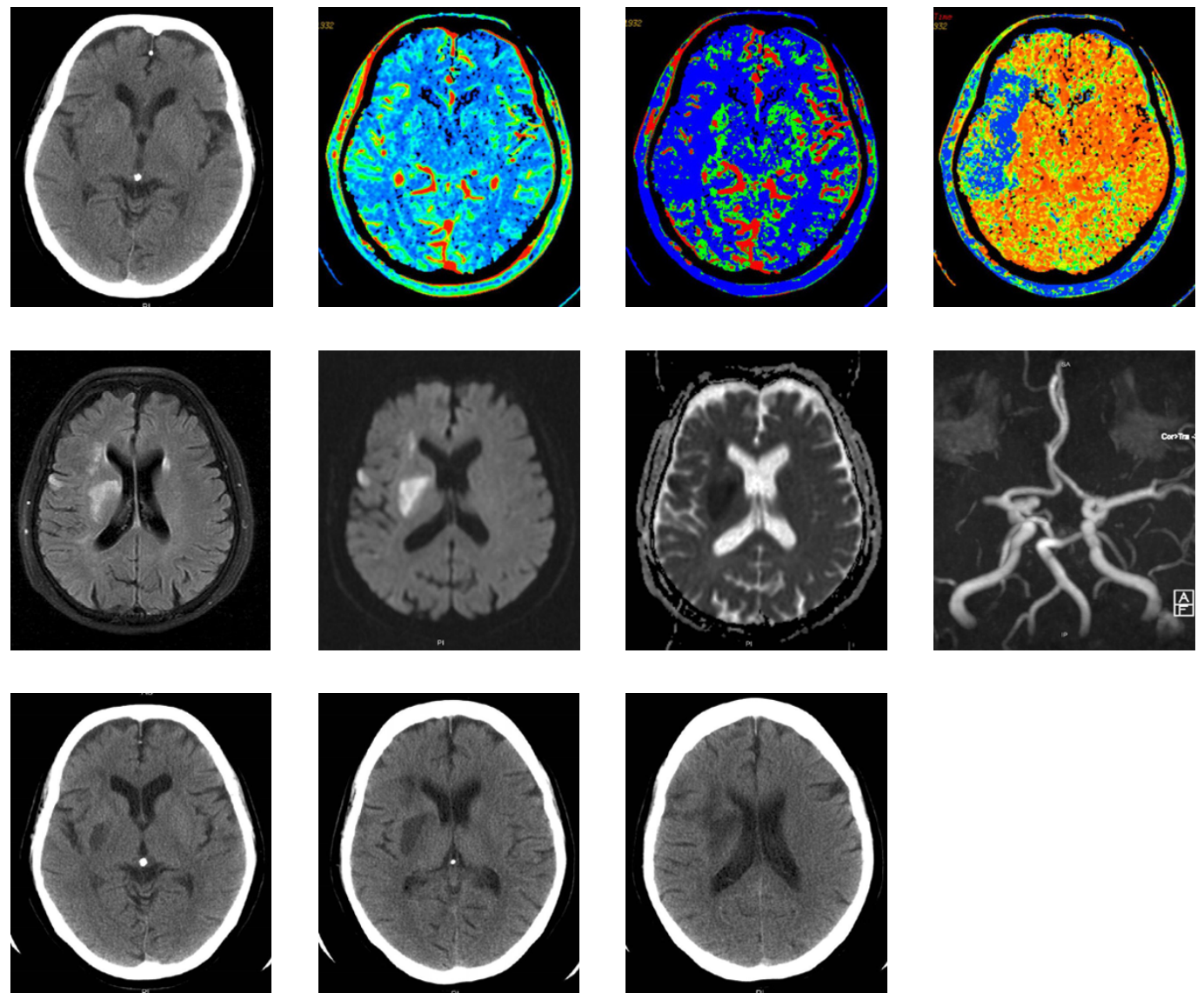

\section{Figure 2}

Top row, left: Admission CT showing hypoattenuation of the right lentiform nucleus and insular cortex as well as slight swelling of cortical sulci in the right frontoparietal region. Top row, the third and the fourth pictures: The perfusion CT on admission, with flow and mean transit time CT showing a broad perfusion deficit in the right MCA region. Top row, the second picture: The volume chart shows only a small deficit in the right MCA region, consistent with the presence of a large ischemic penumbra in the cortical cerebral tissue. Middle row: The follow-up brain MRI showing the presence of a limited subcortical infarct on the distal region of the right lentiform nucleus and corona radiata. Signs of only a subtle cortical infarction are seen. From left to right: FLAIR MRI, T2-diffusion-weighted MRI, apparent diffusion coefficient. Middle row, right: The persistence of the right MCA occlusion on the follow up MRA. Bottom row: Day 2 CT showed that the extent of the subcortical infarct was substantially smaller than that of the perfusion deficit found in the CT perfusion brain scan obtained on admission.

cially in cases where BP drops during the first night following the admission. In accordance with previous reports of using $\mathrm{IH}[9]$ this study demonstrates relative safety of PHE- IH, the patient was closely monitored, and no evidence of cardiac ischemia were seen, the chest $x$ ray did not reveal any signs of pulmonary edema, and no hemorrhagic transformation on CT scans was observed.

In conclusion, PHE-IH seems to be feasible and could be beneficial in selected stroke patients within 6 hours from stroke symptom onset. A prospective, randomized study of PHE-IH could be warranted to examine whether PHE-
IH can salvage ischemic brain tissue in acute, documented cerebrovascular occlusions that are not amenable for thrombolytic therapy even though ischemic penumbra still exists.

\section{Abbreviations}

MCA-middle cerebral artery

MAP-mean arterial pressure

BP-blood pressure 
IH-induced hypertension

PHE-phenylephrine

NIHSS-National Institute of Health Stroke Scale

PHE-IH phenylephrine-induced hypertension

CBF-cerebral blood flow

CAD-coronary artery disease

AF-atrial fibrillation

mRS-modified Rankin scale

\section{Competing interests}

The author(s) declare that they have no competing interests.

\section{Authors' contributions}

TB drafted the manuscript and made a contribution to design, analysis and acquisition and interpretation of data. PL made a contribution to design and conception of the study and participated in the analysis of data, and revised the manuscript that led to the final approval of the current submission. $\mathrm{OH}$ and $\mathrm{OS}$ were involved in the acquisition of clinical data and in the interpretation of data.

\section{Acknowledgements}

Written consent was obtained from the patient for publication of this study.

\section{References}

I. Broderick J, Brott T, Barsan W, Haley EC, Marler J, Sheppard G, Blum $\mathrm{C}$ : Blood pressure during the first minutes of focal cerebral ischemia. Ann Emerg Med 1993, 22: I438-1443.

2. Mattle HP, Kappeler L, Arnold M, Fisher U, Nedeltchev K, Remonda $\mathrm{L}$, Jakob S, Schroth G: Blood pressure and vessel recanalisation in the first hours after ischemic stroke. Stroke 2005, 36:264-269.

3. Eames PJ, Blake MJ, Dawson S, Panerai RB, Potter JF: Dynamic cerebral autoregulation and beat-to-beat blood pressure control are impaired in acute ischemic stroke. JNNP 2002, 72:467-472.

4. Lindsberg PJ: High blood pressure after acute cerebrovascular occlusion: risk or risk marker? Stroke 2005, 36:268-269.

5. Hillis AE, Ulatowski JA, Barker PB, Torbey M, Ziai W, Beauchamp NJ, Wityk RJ: A pilot randomized trial of induced blood pressure elevation: effects on function and acute perfusion in acute and subacute stroke. Cerebrovasc Dis 2003, 16:236-246.

6. Rordorf G, Koroshetz WJ, Ezzeddine MA, Segal AZ, Buonnanno F: A pilot study of drug induced hypertension for treatment of acute stroke. Neurology 200I, 56:1210-1213.

7. Chalela J, Dunn B, Todd J, Warach S: Induced hypertension improves cerebral blood flow in acute ischemic stroke. Neurology 2005, 64:1979.

8. Darby DG, Barber PA, Gerraty RP, Desmond PM, Yang Q, Parsons $\mathrm{M}$, Li T, Tress BM, Davis S: Pathophysiological topography of acute ischemia by combined diffusion-weighted and perfusion MRI. Stroke 1999, 30:2043-2052.

9. Koenig MA, Geocadin RG, Grouchy M, Glasgow J, Vimal S, Restrepo L, Wityk R: Safety of induced hypertension therapy in patients with acute ischemic stroke. Neurocrit Care 2006, 04:3-7.

\section{Pre-publication history}

The pre-publication history for this paper can be accessed here:

http://www.biomedcentral.com/1471-2377/6/46/prepub

\section{Publish with Biomed Central and every scientist can read your work free of charge}

"BioMed Central will be the most significant development for disseminating the results of biomedical research in our lifetime. "

Sir Paul Nurse, Cancer Research UK

Your research papers will be:

- available free of charge to the entire biomedical community

- peer reviewed and published immediately upon acceptance

- cited in PubMed and archived on PubMed Central

- yours - you keep the copyright

Submit your manuscript here:

http://www.biomedcentral.com/info/publishing_adv.asp
BioMedcentral 